\title{
Spontaneous cerebrospinal fluid leak at the clivus
}

\author{
Krzysztof Oleś ${ }^{1,2}$, Jacek Składzien ${ }^{1}$, Marek Betlej ${ }^{3}$, Robert Chrzan ${ }^{4}$, Joanna Mika ${ }^{5}$ \\ ${ }^{1}$ Department of Otolaryngology, Jagiellonian University, Medical College, Krakow, Poland \\ ${ }^{2}$ Department of Otolaryngology and Reconstructive Surgery, Institute of Oncology Gliwice Branch, The Maria Sklodowska-Curie \\ Memorial Cancer Center, Gliwice, Poland \\ ${ }^{3}$ Department of Neurosurgery, Institute of Neurology, Jagiellonian University, Medical College, Krakow, Poland \\ ${ }^{4}$ Radiology Department, Jagiellonian University, Medical College, Krakow, Poland \\ ${ }^{5}$ Department of Pain Pharmacology, Institute of Pharmacology, Krakow, Poland
}

Videosurgery Miniinv 2015; 10 (4): 593-599

DOI: $10.5114 /$ wiitm.2015.55676

\begin{abstract}
We present a case report of a 60-year-old woman with a spontaneous cerebrospinal fluid leak at the clivus, obesity and no history of trauma. Follow-up imaging scans confirmed enlargement of the defect within the posterior clival framework to the size of $16 \times 9 \times 4 \mathrm{~mm}$ with a suspected meningocerebral hernia. The surgeons used the "two nostrils -four hands" endoscopic operating technique. The patient reported a history of cerebrospinal fluid leaks lasting for 3 years, with increasingly shorter leak-free periods and an increasing incidence of inflammatory complications. The patient recovered without complications, and she was discharged 14 days after the surgery. Good local outcome and improved patient condition were achieved postoperatively.
\end{abstract}

Key words: spontaneous cerebrospinal fluid leak, clivus, endoscopic sinus surgery, benign intracranial hypertension, four hands endoscopic operating technique.

\section{Introduction}

Idiopathic or spontaneous cerebrospinal fluid leaks comprise $5-10 \%$ of all fluid leaks depending on the accepted criteria [1-3]. Elevated intracranial pressure (ICP) likely causes spontaneous cerebrospinal fluid leaks. The most important indication for surgical management of fluid leak is cerebrospinal meningitis or other severe complications, either intracranial or, less frequently, orbital $[1,4]$.

Persistently elevated intracranial pressure characterizes benign intracranial hypertension (BIH). Accompanying symptoms include chronic headaches, optic disc swelling, disturbed vision, cranial nerve palsy, periodical or continuous cerebrospinal fluid leaks and cerebrospinal meningitis [4]. The disorder is most common in young obese women. Predispos- ing factors include chronic steroid therapy, chronic antibiotic therapy (tetracyclines) and systemic diseases, such as systemic lupus erythematosus (SLE), chronic renal diseases and hypervitaminosis $A[1,5]$. Causes of disturbed cerebrospinal fluid production include disturbances in spontaneous cerebrospinal fluid (CSF) production and absorption, cerebral edema, endocrine disorders and intracranial hypertension Studies also confirmed intracranial venous flow obstruction as the cause of increased ICP [5]. Some studies report that elevated ICP results from reduced extracranial venous flow. Elevated ICP weakens the natural skull base barrier within the subarachnoid cavity, which exposes a potential leak route. Normal intracranial pressure is observed in $55 \%$ of case [1]. Benign intracranial hypertension is one cause of slow skull base erosion. Erosion is also caused by

Address for correspondence

Krzysztof Oleś MD, PhD, Department of Otolaryngology and Reconstructive Surgery, Institute of Oncology Gliwice Branch,

The Maria Sklodowska-Curie Memorial Cancer Center, 15 Wybrzeże Armii Krajowej St, 44-101 Gliwice, Poland, phone: +48 502080238 , +48 3227884 17, e-mail: olokrista@op.pl 
the disturbed production and absorption of cerebrospinal fluid. A cough, sneeze, or physical effort leads to CSF leak manifestations in all of these cases. The diagnostic process involves 3D reconstruction of computed tomography (CT) scans, magnetic resonance imaging (MRI) and CT cisternography, which determines the diagnosis, surgical procedure plan and potential deviations in intracranial anatomy [6].

\section{Case report}

A 60-year-old woman presented with grade 3 (morbid) obesity, body mass index (BMI) $40.02 \mathrm{~kg} / \mathrm{m}^{2}$,
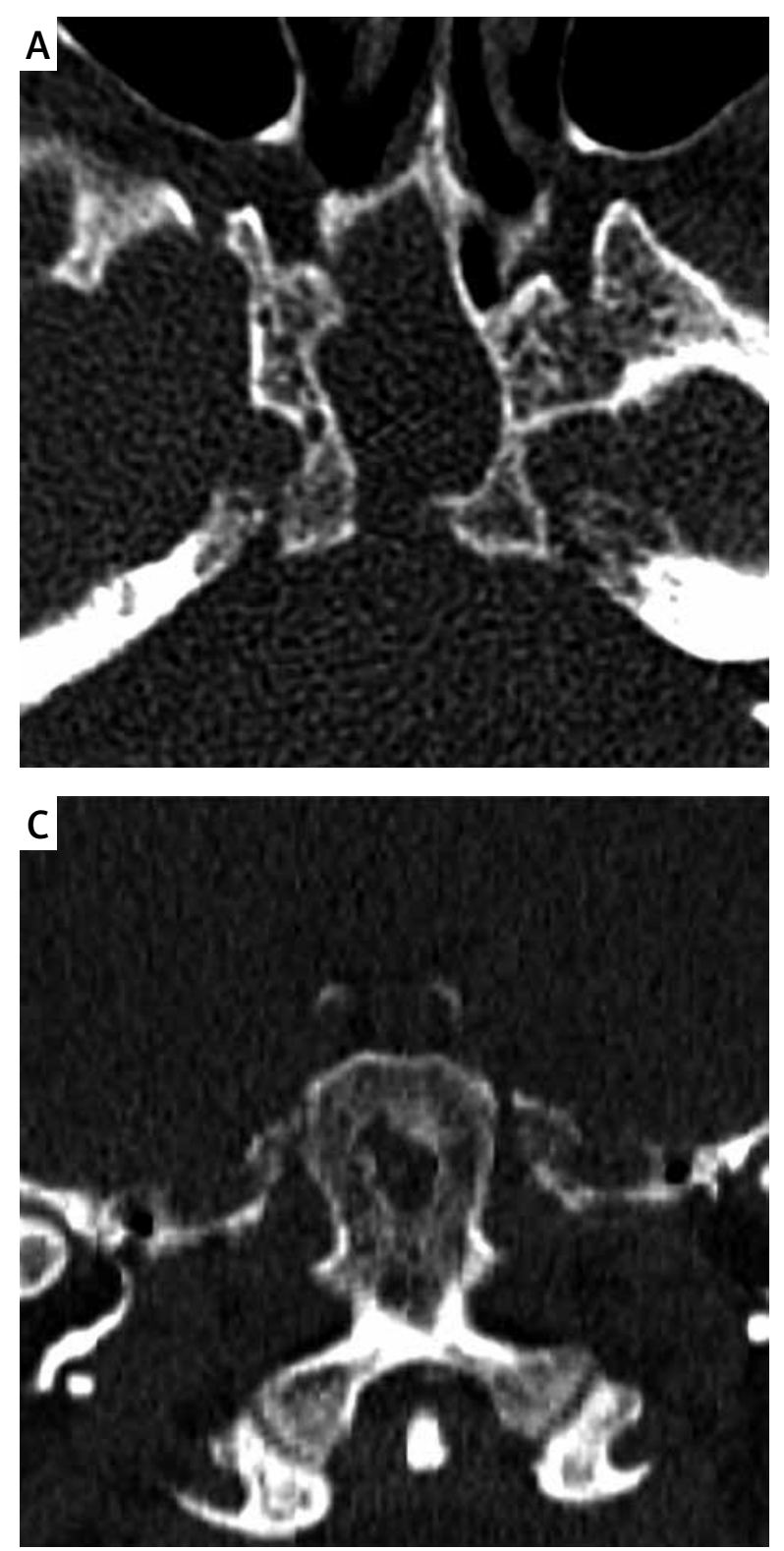

body weight $108 \mathrm{~kg}$, and height $164 \mathrm{~cm}$. Patient-reported history included venous insufficiency and two operations for varicose veins in the lower limbs. The patient underwent a hysterectomy due to giant sarcomas, and a history of two childbirths by natural labor. She was not treated for other systemic diseases. The first rhinorrhea occurred approximately 3 years before. Initially, her symptoms were believed to be due to chronic allergic rhinitis. The patient experienced more frequent upper respiratory tract infections accompanied by pronounced general symptoms, an elevated body temperature of $38-39^{\circ} \mathrm{C}$, fatigue, muscle and joint pains, altered mood and meningeal irrita-

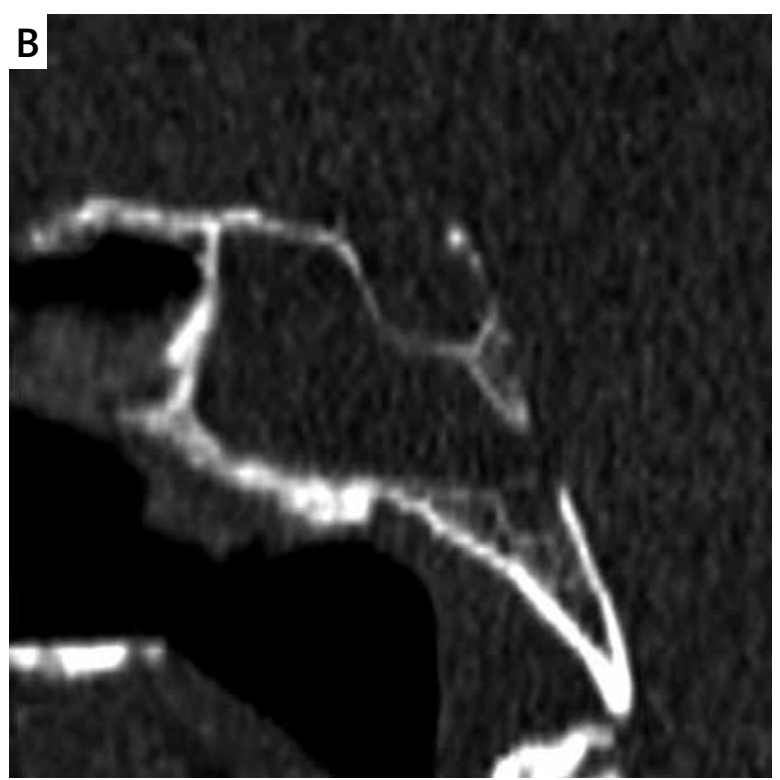

Photo 1. Computed tomography images before surgery, bone kernel/window: A - axial, B - sagittal reconstruction, $\mathrm{C}$ - oblique reconstruction in the plane of the clivus. An irregular canal in the clivus connects the right sphenoid sinus with the posterior cranial fossa 
tion. The patient was referred to a laryngology department at her place of residence where CT and MRI diagnostics revealed a clival bone defect of $9 \times 7 \mathrm{~mm}$ and $8 \times 5 \times 6 \mathrm{~mm}$, which was subsequently diagnosed and differentiated as cerebrocranial hernia. Nasal cerebrospinal fluid leak was confirmed in biochemical investigations. The patient was hospitalized in our clinic in July 2014 due to sinogenic orbital complications that manifested as swelling and congestion of the frontal orbital segment and irritation of cerebrospinal meninges. Imaging diagnostic scans confirmed a bone defect within the posterior clival framework that was larger compared to earlier scans. Fluid leaks occurred at several-day intervals. Therefore, the patient declined consent for the procedure and underwent consultations at other neurosurgery clinics. She received no guarantees of the potential endoscopic management of the leak. An intracranial approach was proposed. The patient was referred to our clinic for the second time due to increasing headaches, intense, continuous rhinorrhea, irritation of cerebrospinal meninges with inflammatory orbital complications and oral herpes of the upper lip. Follow-up imaging scans confirmed enlargement of the defect within the posterior clival framework to the size of $16 \times 9 \times 4 \mathrm{~mm}$ with a suspected meningocerebral hernia (Photos $1 \mathrm{~A}-\mathrm{C}$ ). In $\mathrm{CT}$ and $\mathrm{MRI}$ performed three times before surgery, pathology, especially hydrocephalus, in the central nervous system was excluded by the radiologist (Photos $2 \mathrm{~A}, \mathrm{~B}$ ).

An intranasal transsphenoid endoscopic exploration of the skull base defect and fluid leak closure procedure was performed using DigiPointer (Collin) neuronavigation with neurosurgical assistance. The procedure started with a complete bilateral sphenoethmoidectomy and radical resection of the vomer. This step allowed the surgeons to use the two nostrils - four hands operating technique. The next step identified the Turkish saddle, optic nerve, and carotid arteries on both sides within the sphenoid sinus. An extensive defect in the bone framework of the left internal carotid artery and exposed meninx within the sphenoid sinus were revealed, as observed previously in CT scans. The anterior and middle portions of the clivus were exposed between both carotid arteries. Resection of the barrier between both sides of the sphenoid sinus facilitated visualization of the bone defect within the posteroinferior part of the left sphenoid sinus and the posterior wall of the superior and middle clivus (Photos $3 \mathrm{~A}-\mathrm{C}$ ). The bony edges of the defect framework and the critical structure, in this case the basal artery, were identified during the procedure (Photos $4 \mathrm{~A}, \mathrm{~B}$ ). The artery protruded into the clival defect, and it was shifted $2 \mathrm{~mm}$ to the
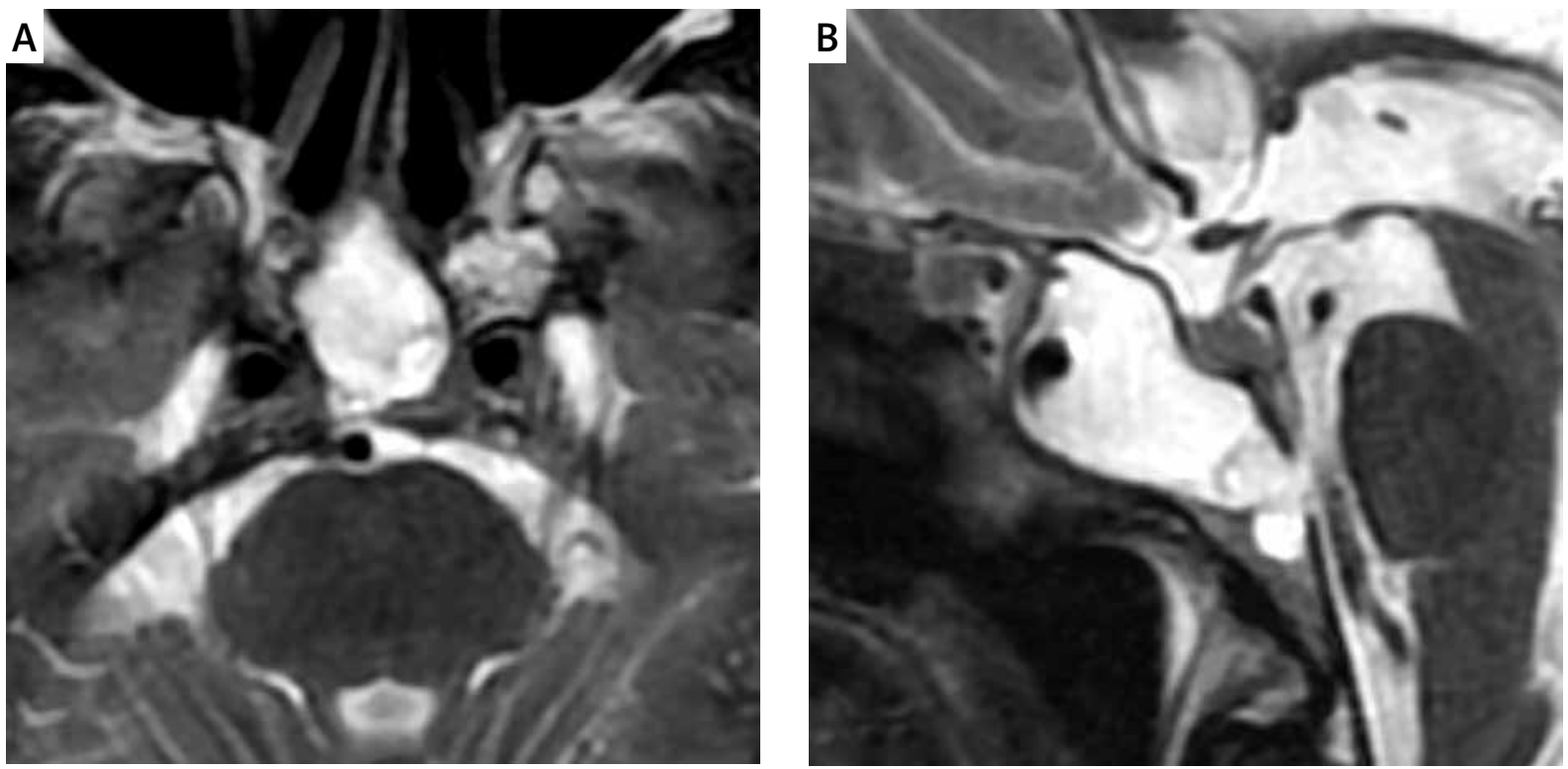

Photo 2. Magnetic resonance images before surgery, T2-weighted sequences: A - axial, B - sagittal. An irregular canal in the clivus connecting the right sphenoid sinus with the posterior cranial fossa, the right sphenoid sinus filled with cerebrospinal fluid, and the basilar artery near the canal, which increases the surgical risk considerably 

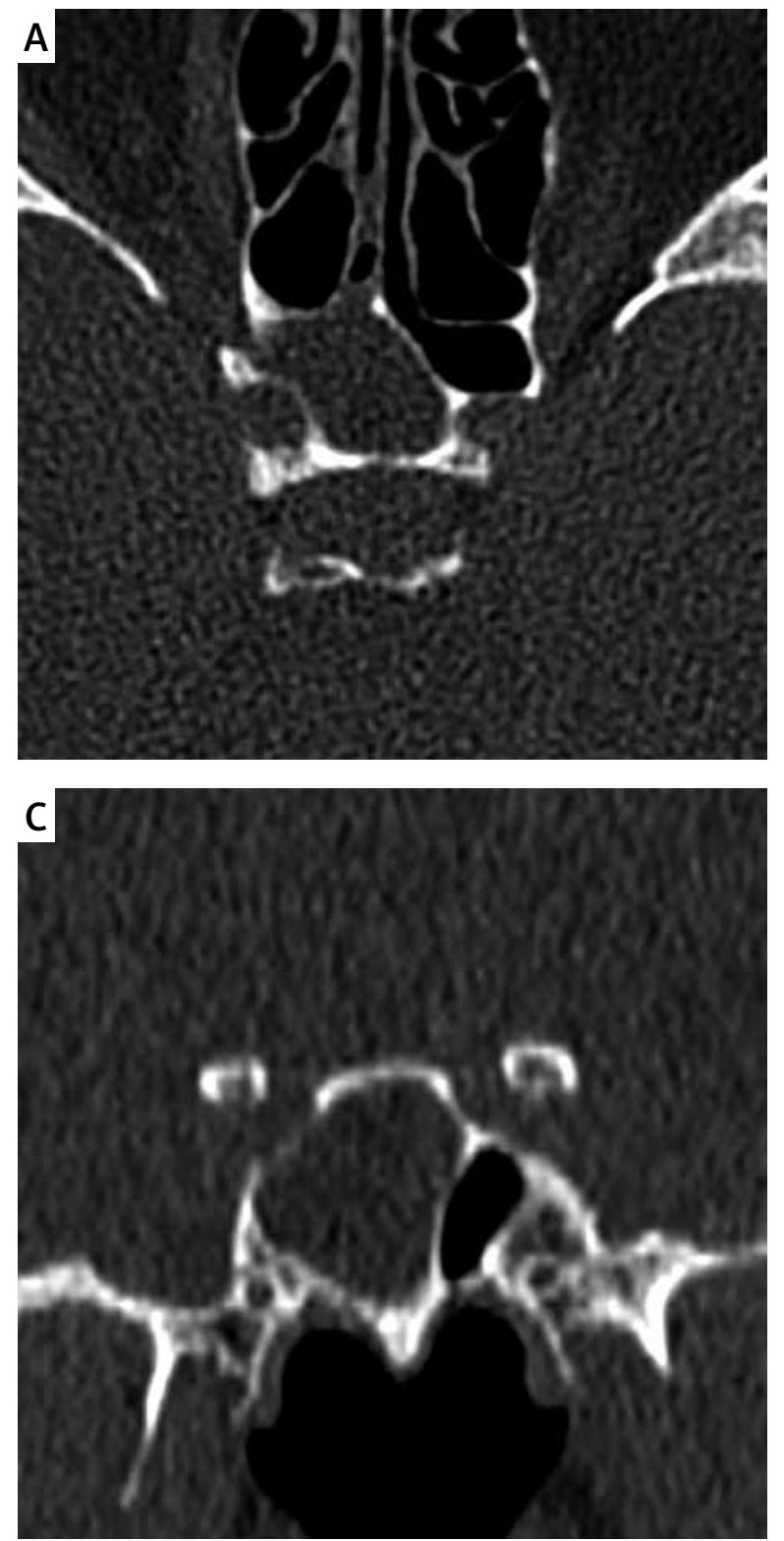

right of the medial line, as observed in the MRI scan. The bony edges of the defect were carefully dissected, and fragments of the cerebrospinal meninges were located intracranially with much difficulty (Photos 5 A-D). The skull base defect was repaired using fascia lata, septal nasal cartilage, autogenic fat, vastus lateralis muscle, fast-binding adhesive (fibrin glue) and a Tisseel TachoSil fibrin sealant patch.

High-pressure cerebrospinal fluid leak and local bleeding were encountered intraoperatively despite hypotension. Lumbar constant drainage $(\phi 17 \mathrm{G})$ into the $L_{3-4}$ space was used intraoperatively. Postoperative measurement of CSF pressures with a lumbar

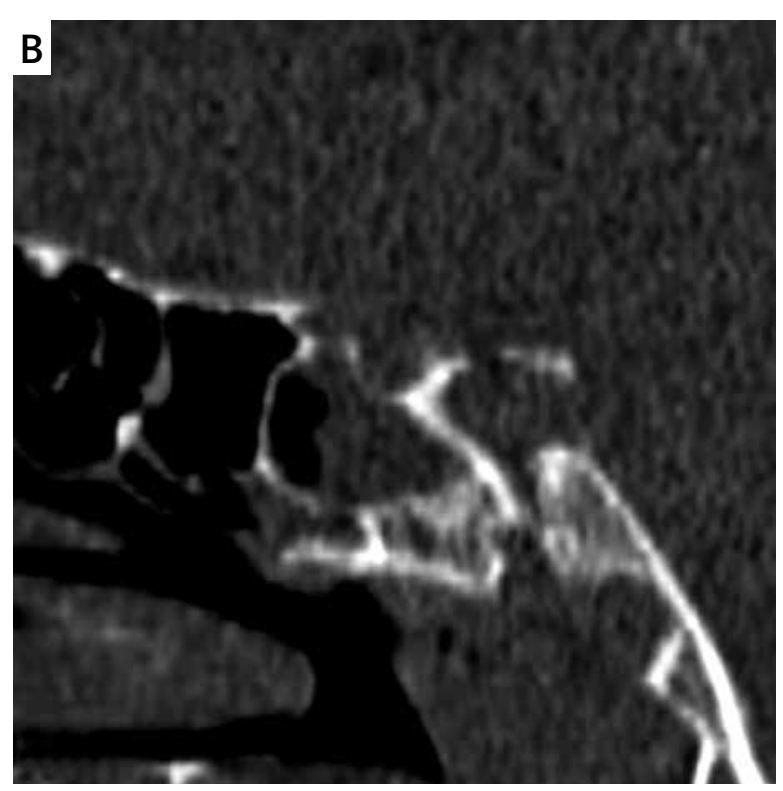

Photo 3. Computed tomography images before surgery, bone kernel/window: A - axial, B - sagittal reconstruction, $\mathrm{C}$ - coronal reconstruction. A very thinned wall of the right sphenoid sinus at the site of the right internal carotid artery pressing into the sinus, which additionally increases the surgical risk

drain showed a mean of $30.0 \mathrm{~cm} \mathrm{H}$ O. The patient was awakened from anesthesia 3 days after the procedure. A follow-up head CT scan 7 days after surgery revealed no intracranial complications or neurological defects. The patient has remained free of clinical features of CSF leaks postoperatively. Perioperative vancomycin, metronidazole and meropenem were used for 14 days because Pseudomonas aeruginosa and Klebsiella pneumoniae bacilli were detected in microbial investigations. Lumbar drainage was used 1 to 7 days after surgery. CSF loss levels varied around approximately $150 \mathrm{ml}$ per day, except for day 3 when the patient was transferred from the ICU to the in- 

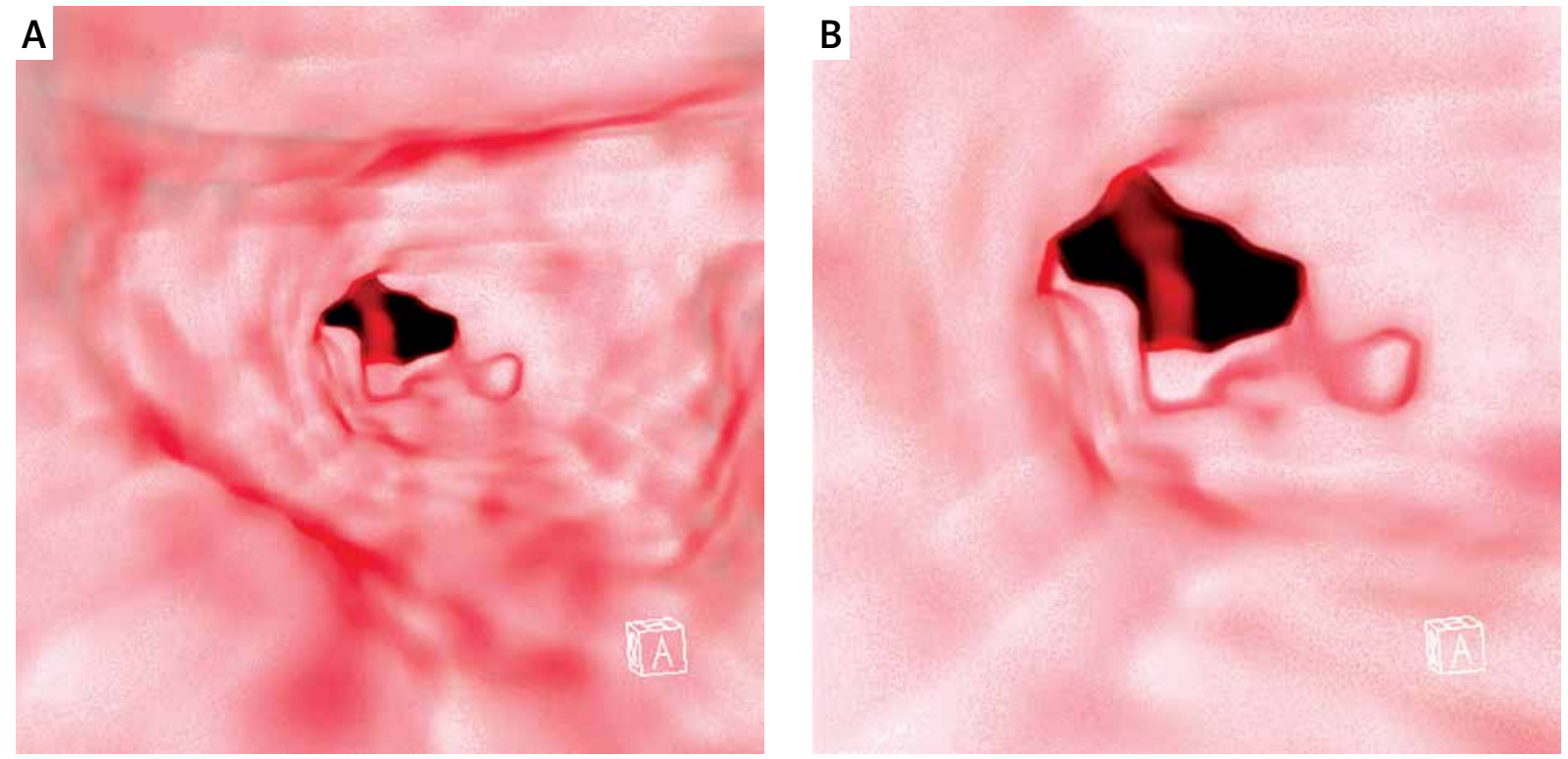

Photo 4. Virtual endoscopy reconstructions from CT images before surgery: A - view from inside the right sphenoid sinus, B - view into the canal in the clivus. The basilar artery is visible near the canal, which increases the surgical risk considerably

creased postoperative supervision room, and the total leak volume was $350 \mathrm{ml}$. The patient was in limited neurological contact and required medications to improve intracranial flow on this day. The patient has recovered without complications, and she was discharged 14 days after the surgery.

\section{Discussion}

A survey of English-language PubMed entries published until December 2014 was performed using the following keywords: "increased intracranial pressure", "cerebral hernia", "skull base defect", "spontaneous cerebrospinal fluid leak", "rhinorrhea" and "otorrhea". No documented cases of spontaneous CSF leak within the clivus region were found. Spontaneous rhinorrhea is a rare pathological condition that is associated with high diagnostic and therapeutic difficulties. It poses a significant therapeutic challenge to surgeons treating skull bone disorders. At the clivus spontaneous CSF leak can be secondary to ecchordosis physaliphora, which should be taken into consideration in differentiation.

Rhinorrhea is closely associated with $\mathrm{BIH}[1,5]$. A ventriculoperitoneal shunt remains the standard method of $\mathrm{BIH}$ treatment in patients with spontaneous nasal CSF leak [5]. We did not stick to this procedure (ventriculoperitoneal shunt) as an intracranial mass was not suspected in radiological ex- amination, the ICP level was known after the surgery, and twice there was meningitis in the last 6 months. Determination of the defect location in relation to critical structures, including the course of carotid arteries within the intracavernous segment, vertebral arteries and basal artery in relation to the defect, is crucially important. Transcranial, transsphenoid, oropharyngeal and maxillary osteotomy approaches are potential methods to access the region of interest. Transcranial approaches require cerebral retraction, which increases the risk of brain swelling, hematoma and injuries of the internal carotid artery, basal artery and the optic nerve. The use of anterior approaches, namely transnasal, transoral and transfacial approaches, may significantly reduce these complications [7-10]. Endoscopic surgery creates a new method for the management of spontaneous CSF leaks within the clivus region and facilitates good visualization of critical structures [2].

Surgeons consider patients with spontaneous fluid leak and elevated ICP to be among the most difficult surgical cases. The rates of repeat surgery following endoscopic approach procedures are estimated at $21-87 \%$. Bifrontal craniotomy using autogenic tissues for reconstruction of skull base defect and meningeal repair should be at least a rescue option for endoscopic surgery. The two nostrils - four hands technique, accompanied by increasingly ad- 

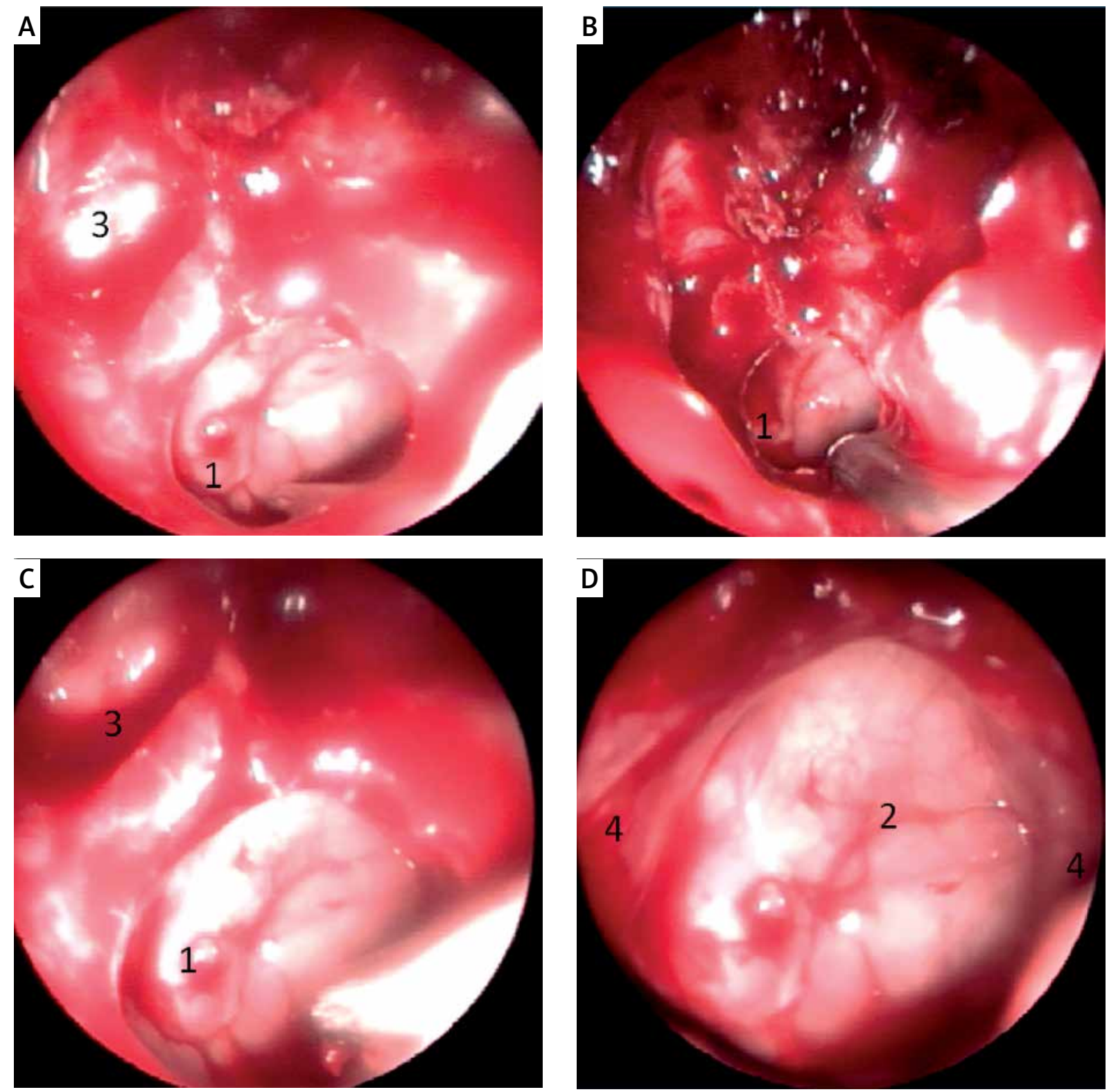

Photo 5. Intra-operative endoscopic view ( $30^{\circ}$ scope) of a patient who presented with CSF spontaneous leak. Note the relationship between the defect, basilar artery and paraclival internal carotid artery

1 - basilar artery, 2 - spinal cord, 3 -sphenoid sinus, 4-internal carotid artery.

vanced instrumentation, allows the efficient management of spontaneous CSF leaks [8].

Intracranial approaches are widely accepted as the correct methods. Intranasal endoscopic approaches should be used to repair small defects within the bone framework because they help reduce complications [2, 7]. Failures in the use of endoscopic approaches may be due to the management of leaks in an inappropriate location, the use of inappropriate grafts, or graft translocation. It is extremely important to identify the bony edges surrounding the defect and gradually identify critical structures (the basal artery in this case) within the vicinity during exploratory endoscopic procedures $[2,7,8]$.

In conclusion, spontaneous CSF leaks require invasive examinations to determine the cause of the leak. Patients with spontaneous rhinorrhea 
are at a higher risk of complications compared to patients after trauma or surgery. The presence of $\mathrm{BIH}$ requires diagnostic tests and treatment. Examinations include the diagnostics of the extracranial venous systems, which may be responsible for elevated ICP, and these examinations should be included in the perioperative plan. Lumbar drainage, acetazolamide and permanent separation of fluid leaks may reduce the failure risk in endoscopic procedures. We presented this case because of the very rare location of the skull base framework defect and the associated difficulties in the surgical management of fluid leak using endoscopic techniques.

\section{Conclusions}

We described this case since it is a very rare and difficult defect located in the skull base framework - a female patient presented with grade 3 (morbid) obesity, BMI $40.02 \mathrm{~kg} / \mathrm{m}^{2}$ with a clival bone defect of $9 \times 7 \mathrm{~mm}$ and $8 \times 5 \times 6 \mathrm{~mm}$. Follow-up imaging scans revealed enlargement of the defect within the posterior clival framework (size $16 \times 9 \times 4 \mathrm{~mm}$ ) with a suspected meningocerebral hernia or possibly secondary to ecchordosis physaliphora, which should be taken into the consideration in differentiation. We presented the associated complications in the surgical management of fluid leak using endoscopic techniques.

\section{Conflict of interest}

The authors declare no conflict of interest.

\section{References}

1. Bledsoe JM, Moore EJ, Link MJ. Refractory cerebrospinal fluid rhinorrhea secondary to occult superior vena cava syndrome and benign intracranial hypertension: diagnosis and management. Skull Base 2009; 19: 279-85.

2. Muscatello L, Lenzi R, Dallan I, et al. Endoscopic transnasal management of cerebrospinal fluid leaks of the sphenoid sinus. Craniomaxillofac Surg 2010; 38: 396-402.

3. Pérez MA, Bialer OY, Bruce BB, et al. Primary spontaneous cerebrospinal fluid leaks and idiopathic intracranial hypertension. J Neuroophthalmol 2013; 33: 330-7.

4. Verma N, Savy LE, Lund VJ, et al. An important diagnosis to consider in recurrent meningitis. JRSM Short Rep 2013; 4 2042533313486640

5. Van Zele T, Kitice A, Vellutini E, et al. Primary spontaneous cerebrospinal fluid leaks located at the clivus. Allergy Rhinol (Providence) 2013; 4: e100-4.
6. Jeswani S, Gupta S, Hagiwara M, Lebowitz RA. Non-invasive radiologic evaluation of intermittent CSF rhinorrhea. J Otol Rhinol 2013; $2: 3$.

7. Virk JS, Elmiyeh B, Saleh HA. Endoscopic management of cerebrospinal fluid rhinorrhea: the charing cross experience. J Neurol Surg B Skull Base 2013; 74: 61-7.

8. Snyderman CH, Carrau RL, Kassam AB, et al. Endoscopic skull base surgery: principles of endonasal oncological surgery. J Surg Oncol 2008; 97: 658-64.

9. Cassano M, Felippu A. Endoscopic treatment of cerebrospinal fluid leaks with the use of lower turbinate grafts: a retrospective review of 125 cases. Rhinology 2009; 47: 362-8.

10. Komatsu M, Komatsu F, Cavallo LM, et al. Purely endoscopic repair of traumatic cerebrospinal fluid rhinorrhea from the anterior skull base: case report. Neurol Med Chir (Tokyo) 2011; 51: 222-5.

Received: 27.07.2015, accepted: 24.09.2015. 